Service social

\title{
Une grille d'évaluation systémique des familles recomposées
}

\section{Denise Robitaille et Christian Côté}

Volume 39, numéro 3, 1990

Familles recomposées après divorce

URI : https://id.erudit.org/iderudit/706505ar

DOI : https://doi.org/10.7202/706505ar

Aller au sommaire du numéro

\section{Éditeur(s)}

École de service social de l'Université Laval

\section{ISSN}

1708-1734 (numérique)

Découvrir la revue

\section{Citer cet article}

Robitaille, D. \& Côté, C. (1990). Une grille d'évaluation systémique des familles recomposées. Service social, 39(3), 135-156. https://doi.org/10.7202/706505ar

\section{Résumé de l'article}

L'article présente de façon détaillée une grille organisant les données souvent fort complexes recueillies auprès des familles recomposées. Il s'adresse aux intervenants. Le cadre théorique, systémique et développemental est présenté brièvement, et la grille est appliquée à une situation. Les différents sous-systèmes sont établis de façon précise, en distinguant ce qui relève de la famille recomposée et des étapes de sa formation et ce qui relève des personnes reliées à la famille, mais externes à sa structure. Les différentes fonctions des sous-systèmes sont clarifiées, avec leurs modalités de réalisation. La grille débouche aisément sur la formulation claire du problème à travailler, de l'objectif à atteindre et de l'engagement de chacun. Enfin, les auteurs présentent brièvement les principales attitudes et techniques qui permettent d'appliquer la grille de façon rapide et efficace. 
Denise Robitaille, travailleuse sociale au Centre de consultation conjugale et familiale de Québec.

Christian Côté, professeur à l'École de service social de I'Université Laval.

\section{Une grille d'évaluation systémique des familles recomposées}

\section{Denise Robitaille Christian Côté}

Depuis plusieurs années, dans notre travail de formation à l'intervention planifiée à court terme, constatant que la multitude des données recueillies dans l'exploration rendait difficile l'élaboration d'un plan précis d'intervention, nous avons bâti des grilles d'analyse qui permettent d'organiser ces données, qu'il s'agisse d'une personne, d'un couple ou d'une famille. Nous avons alors constaté que lorsque nous rencontrions des familles recomposées, la grille d'évaluation familiale ne suffisait pas, mais que les mêmes éléments se répétaient, avec des variations d'après les personnes et les familles. Nous avons développé une grille adaptée aux caractéristiques de ces familles où les sous-systèmes sont plus complexes et plus nombreux. L'article qui suit présente cette grille d'évaluation. L'analyse théorique des familles recomposées ayant été présentée dans les articles précédents de cette publication, nous nous orienterons, après un rappel des concepts utilisés, vers les applications concrètes. L'article doit donc être lu en tenant compte de ces articles théoriques, et s'adresse surtout aux praticiens qui ont à travailler avec ces familles et se sentent débordés par la somme des données apparemment non conciliables. Nous ne présenterons pas les bases théoriques et méthodologiques de l'intervention planifiée à court terme, décrites ailleurs'.

\section{Présentation de la grille}

Comme les grilles portant sur les personnes, les couples et les familles que nous avons développées pour les programmes de forma- 
tion au Centre de consultation conjugale et familiale de Québec, celle-ci dégage, dans un cadre d'analyse systémique, les données les plus significatives parmi l'ensemble de ce qui a été recueilli durant l'exploration et facilite, en en faisant ressortir la pertinence, une planification plus exacte, mieux structurée de l'intervention. Les données non retenues sont consignées dans les notes au dossier. La grille porte ici sur trois pages; son contenu est surtout évaluatif, faisant ressortir les forces et les faiblesses du système-client dans sa structure et son fonctionnement. Quelques données factuelles y sont intégrées, pour situer les étapes d'évolution de la famille. Le cadre théorique utilisé repose d'abord sur l'analyse systémique de Minuchin ${ }^{2}$, pour la structure familiale et les fonctions des sous-systèmes; pour I'identification de la hiérarchie et des patterns transactionnels, ainsi que pour la fonction positive des symptômes, nous nous référons à Haley et Madanès ${ }^{3}$.

Un survol des deux premières pages de la grille (p. 142 et 143) nous indique que nous devons d'abord connaître les composantes du système concerné. II s'agit des membres de la famille qui vivent habituellement ou fréquemment sous le même toit; ce sont aussi les autres membres des familles dont ces personnes faisaient autrefois partie ainsi que les personnes importantes dans la situation et qui sont encore en relation étroite avec les membres.

Nous déterminons le fonctionnement des membres dans les différents sous-systèmes, en prêtant attention aux rôles propres à chaque sous-système. Une fois ces rôles définis, il est nécessaire de distinguer clairement entre les rôles des conjoints, qui ne sont pas parents des mêmes enfants, et ceux des parents, qui ne sont plus conjoints. Il en est de même pour les enfants, qui forment des soussystèmes différents à l'intérieur de la fratrie. La grille identifie les activités complémentaires des parents, dont l'un est absent de la famille recomposée mais dont l'autre est présent, et les nouveaux rôles des conjoints dont un n'est pas parent, mais est un adulte occupant une position de type parental. Ici nous faisons référence aux fonctions du sous-système parental : le " support ", la " guidance ", le caring, la " réception " et l'écoute des besoins : qui le fait, avec qui, qui est négligé, qui n'est pas protégé ? Qui exprime quels sentiments, à l'adresse de qui ? Nous nous référons ici à l'approche de Maurizio Andolfi ${ }^{4}$ auprès des systèmes rigides dans la résolution des conflits trop menaçants.

Nous illustrons les positions et les frontières ainsi définies avec une carte familiale, en y ajoutant les systèmes extérieurs et les personnes significatives.

Nous définissons l'étape où se trouve la famille dans sa recomposition. Cela s'exprime en termes de dates, de nombre d'années ou 
de mois entre la séparation et la recomposition actuelle. Nous insistons beaucoup sur ces données factuelles, trop souvent oubliées et qui sont pourtant nécessaires, car nous croyons que les familles doivent passer par certaines étapes, rappelées plus haut dans l'article de Gerry Marino et Francine Fortier. L'oubli de ces faits peut amener les intervenants à poser des actions valables en soi, mais inefficaces et même nuisibles lorsqu'elles ne sont pas adaptées au moment que vit la famille.

Nous utilisons alors les concepts de deuils et de loyautés, empruntés à Y. Boszormenyi-Nagy ${ }^{5}$ : les deuils de l'union précédente sont-ils réglés ? Y a-t-il des loyautés qui bloquent le passage à l'étape de l'établissement de nouvelles relations? Nous considérons non seulement les enfants, mais aussi les ex-conjoints, que souvent une culpabilité amène à poser des gestes soit déplacés, soit cachés du nouveau conjoint, ce qui entraîne infailliblement des tensions et des querelles. Parfois, nous pouvons faire exprimer des peurs inappropriées chez le nouveau conjoint, qui craint de prendre la place du parent absent, alors que les enfants peuvent en être rendus à désirer que « la blonde du père soit leur amie ${ }^{6}$ ». Les loyautés sont toujours un obstacle important à clarifier lorsque le parent absent vit des difficultés plus grandes que le parent présent. L'expression de ces loyautés ainsi que des culpabilités et des peurs qui les accompagnent peut être difficile et susciter des tensions. Mais ces effets sont moins dommageables à long terme que ce qu'amènent plus tard le secret et l'obligation du silence ressentie par les enfants.

Enfin, dans ces deux premières pages, nous revenons à Minuchin et Haley, pour préciser les frontières et la hiérarchie illustrées dans la carte familiale, en tenant compte de tous les sous-systèmes mentionnés plus haut. Nous décrivons les patterns transactionnels les plus importants, en commençant par les patterns fonctionnels, qui nous serviront pour stimuler les forces de changement, ainsi que les patterns dysfonctionnels qui seront les cibles des corrections.

La grille comprend une autre page, qui présente l'organisation des données vers la préparation de l'action. Nous la présentons ici, car elle est nécessaire même si elle n'offre pas d'éléments propres à la famille recomposée : elle ne comprend pas de faits nouveaux, mais un regroupement des données déjà relevées, pour organiser la planification de l'action. Nous la développerons plus loin dans l'article.

\section{Application de la grille}

Pour mieux comprendre l'utilisation de la grille des familles recomposées, nous en reprenons les différents éléments en les appliquant à un cas type. Les éléments de la grille sont mis en caractères gras, pour les repérer plus facilement. 
L'identification de la famille dit qu'il s'agit de la famille LemieuxLaforce.

Immédiatement après cette identification, nous indiquons la demande de services. II s'agit d'indiquer « qui a demandé quoi ». Si la demande se fait à la suggestion d'une autre personne, ou s'il s'agit $d^{\prime}$ 'une demande de consultation venant directement d'un organisme ou d'un professionnel, il est important de le noter ici, car souvent le fait d'être envoyé par une personne autre que les personnes concernées indique un niveau moindre de motivation. Cet élément donne la demande du client tel que celui-ci perçoit son problème; il ne s'agit donc pas de la situation en général, ni de l'interprétation qu'en fait l'intervenant. Dans une approche à court terme, nous attachons beaucoup d'importance à donner toute l'attention nécessaire à la demande originale, sinon il y a danger de provoquer une forte résistance.

Notre grille révèle :

Demande de services : Marie demande une rencontre familiale parce que sa fille Isabelle ne s'entend pas avec son conjoint Paul.

Dans l'identification et principales caractéristiques, il est important de dresser un bref portrait de chacun, tout en faisant ressortir ce qui le distingue, comme son âge, son occupation, sa scolarité, les points forts ou faibles, les loisirs particuliers, ses problèmes de santé, etc. Ces renseignements pourront être utilisés pour la prescription des tâches ou l'agir de certaines mises en situation.

Nous regroupons dans le a) membres de la famille recomposées les personnes qui vivent ensemble sous le même toit même si certains des enfants n'y demeurent pas à temps plein. Leur présence, à temps partiel, forme une dynamique de famille recomposée. La famille Lemieux-Laforce se compose de :

\section{a) membres de la famille recomposée}

Paul, 40 ans

professeur au Cégep, dépression après divorce, ne prend pas de médicaments, fait beaucoup de conditionnement physique;

Marie, 41 ans diététicienne en centre hospitalier, artiste, chante dans une chorale, plusieurs autres activités à l'extérieur de la maison;

Isabelle, 16 ans fille de Marie, $5^{\mathrm{e}}$ secondaire, tranquille, sort peu, ses résultats scolaires baissent, légèrement obèse;

Frédéric, 11 ans fils de Marie, $5^{\mathrm{e}}$ année, louveteau, fait beaucoup de sports avec son oncle;

Julie, 13 ans fille de Paul, $1^{\text {re }}$ secondaire, garde partagée une semaine sur deux, sportive, compétitions de patinage. 
Sous le b) membres externes mais partageant un sous-système de la famille recomposée, nous indiquons l'ex-conjoint et parent d'enfants de la famille recomposée, donc dans le sous-système parental par rapport à ces enfants. Peuvent également apparaître les enfants nés de ce parent externe, partageant alors un sous-système fraternel avec les enfants de la première union ainsi que la fratrie des enfants de la famille recomposée qui vit à l'extérieur, déjà établie. Ces membres externes sont :

\section{b) membres externes mais dans un sous-système}

Diane, 36 ans

Jean, 45 ans ex-conjointe de Paul, mère de Julie, enseignante au primaire, garde partagée avec Paul;

ex-conjoint de Marie, père d'Isabelle et de Frédéric, ingénieur, travaille à l'extérieur du pays pour de longues périodes, contacts irréguliers avec les enfants;

Benjamin, 18 mois fils de Diane et Pierre, demi-frère de Julie.

Dans la dernière catégorie, c) autres personnes pertinentes, nous retrouvons toutes les autres personnes significatives dans la dynamique de notre système-client, telles que les nouveaux conjoints de parents externes. Ces nouveaux conjoints ne partagent pas de soussystème parental avec les parents de la famille recomposée. Grandsparents, oncles, professeurs, etc., doivent être indiqués si l'intervenant évalue devoir en tenir compte dans son plan d'intervention ou s'ils sont indispensables à la connaissance des personnes en cause. Pour les Lemieux-Laforce, ces gens sont :

\section{c) autres personnes pertinentes}

Pierre, 32 ans conjoint de Diane, père de Benjamin, à sa première union, entraîneur de Julie;

Richard, 36 ans frère de Marie, a deux enfants de l'âge de Frédéric, intègre celui-ci dans ses activités familiales.

Le volet suivant de la grille porte sur les sous-systèmes. Pour le sous-système conjugal, nous nous interrogeons sur le support affectif que les conjoints, non les parents, se donnent tout en précisant si chaque conjoint le fait. II arrive qu'un conjoint est "supportant ", l'autre pas. À l'intimité, nous indiquons la capacité du couple de se donner du temps, des moments privilégiés malgré les obligations de leurs rôles parentaux. Cela est d'autant plus important lorsque la famille est recomposée, n'ayant pas vécu l'étape de vivre à deux sans enfant. Enfin, à la complémentarité, nous commentons si les forces et les faiblesses des conjoints, en tant qu'individus, sont bien équili- 
brées. Ces quelques renseignements recueillis à l'exploration, tant dans le verbal que le non-verbal, sont généralement suffisants pour nous informer de la "bonne santé " ou de la vulnérabilité de ce soussystème conjugal. Dans notre famille type, nous avons :

Sous-système conjugal :

support : très bon pour les deux;

intimité : il s'en donne, en aimerait encore plus;

complémentarité : bonne.

Nous savons donc que le couple est fort et capable de faire face aux situations difficiles. Le problème ne relève pas du sous-système conjugal.

Dans la section suivante, le sous-système parental, la famille recomposée se distingue des familles nucléaires en ce sens qu'elle partage ces sous-systèmes avec les ex-conjoints, Paul partageant son sous-système parental avec Diane, et Marie avec Jean. Les fonctions parentales sont très souvent sources de conflits dans les familles recomposées. Il est donc important de clarifier les rôles de chacun et son habileté à les assumer. Le parent se doit de former une paire exécutive avec son ex-conjoint, l'autre parent qui partage son soussystème parental. Cette paire exécutive se consulte, négocie les décisions majeures à prendre pour leurs enfants et s'accorde dans l'exécution de celles-ci, comme les séjours des enfants chez l'un ou l'autre, l'éducation, les frais financiers, la religion, les vacances, la concordance des loisirs des enfants avec leurs séjours, les anniversaires, les occasions spéciales, l'accès aux grands-parents, etc.

Par ailleurs, ce même parent doit former une autre paire exécutive avec le conjoint actuel, mais sur des plans différents. Cette seconde paire exécutive s'exerce davantage dans l'application des règles du fonctionnement quotidien de la famille recomposée. Bien que le pouvoir décisionnel repose chez le parent, celui-ci doit tenir compte de son conjoint dans l'élaboration des règles; il est souvent nécessaire de négocier, chacun ayant possiblement des styles parentaux différents. Pour former une véritable paire exécutive, le conjoint ne doit pas saboter les règles déterminées par le parent et même les faire respecter. II peut et doit exprimer son opinion sur les sujets importants, mais il doit ensuite accepter les décisions du parent.

L'établissement de ces paires exécutives est l'un des grands défis rencontrés pour les familles recomposées. Système en réorganisation, la famille recomposée met en place ses règles de fonctionnement, vit plusieurs adaptations comme couple et comme parents. Comprendre le fonctionnement de ces paires exécutive permet de mieux assumer tous ces rôles.

Toujours dans le sous-système parental, nous évaluons, pour chaque parent de la famille recomposée, sa capacité de materner, 
guider et contrôler. Par materner, mot utilisé dans son sens large et non pas exclusif aux mères, nous entendons la capacité de répondre aux besoins tant physiques et affectifs des enfants, de les protéger, de répondre à leur besoin de se savoir et de se sentir aimés. Par guider, nous évaluons la capacité de présenter aux enfants des valeurs compatibles avec celles de la société en général, de les pratiquer soimême, de fournir une stimulation appropriée à l'enfant pour actualiser ses capacités et développer son autonomie et sa confiance en lui. Par contrôler, nous faisons référence à la capacité d'assumer l'exercice de l'autorité parentale de façon souple et rationnelle et avec constance, c'est-à-dire mettre des règles adéquates, établir des limites appropriées aux circonstances et prendre les moyens pour les faire respecter. La grille se lit ainsi :

Sous-système parental : Marie

paire exécutive avec ex-conjoint : s.o., trop peu présent;

paire exécutive avec conjoint : en désaccord;

materner : bien;

guider : bien;

contrôler: manque de constance, permissive, délègue trop à Isabelle pour Frédéric.

Sous-système parental : Paul

paire exécutive avec ex-conjoint : bonne;

paire exécutive avec conjoint : en désaccord;

materner : bien;

guider : bien;

contrôler : bien.

Cela nous permet de voir facilement les forces à utiliser et les faiblesses à améliorer chez Marie qui seront intégrées dans le plan d'intervention. Le problème de cette famille recomposée est donc en partie relié à une faiblesse qui n'était pas perçue comme un problème par la famille monoparentale.

Une des particularités des familles recomposées est le nombre et la variété des sous-systèmes fraternels. Nous relevons trois types possibles de sous-systèmes fraternels :

1. sous-système consanguin où la fratrie a en commun les deux parents biologiques;

2. sous-système mi-consanguin où la fratrie a un seul parent biologique;

3. sous-système par alliance où la fratrie n'a en commun aucun parent biologique. 


\section{Carte de la famille recomposée et de ses environnements}

Famille :

Identification et principales caractéristiques

a) membres de la famille recomposée

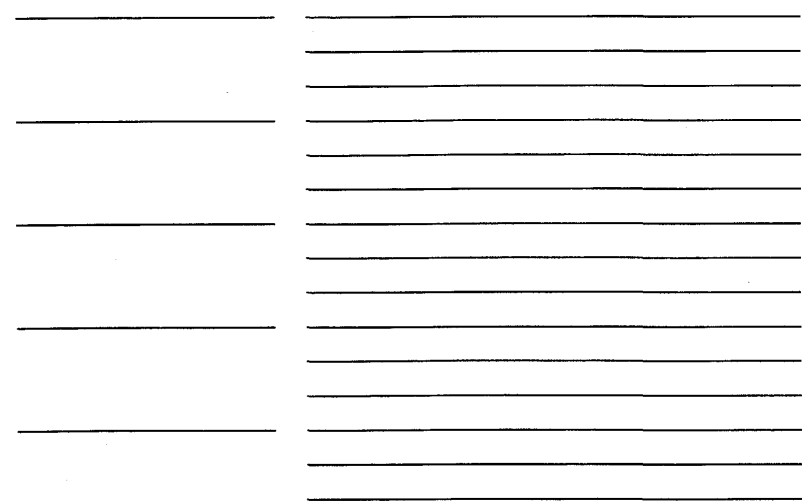

b) membres externes mais dans un sous-système

c) autres personnes pertinentes
Demande de services :

Sous-systèmes :

Conjugal : support

intimité

complémentarité

Parental :

paire exécutive avec ex-conjoint

paire exécutive avec conjoint

materner

guider

contrôler

paire exécutive avec ex-conjoint

paire exécutive avec conjoint

materner

guider

contrôler

Fraternel :

type

laboratoire de vie sociale cohésion

support

type :

cohésion

type :

support

laboratoire de vie sociale cohésion

support 
Séparation :

Deuil :



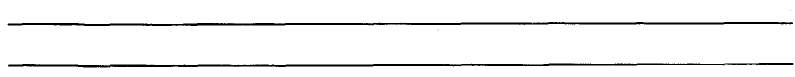

Frontières :
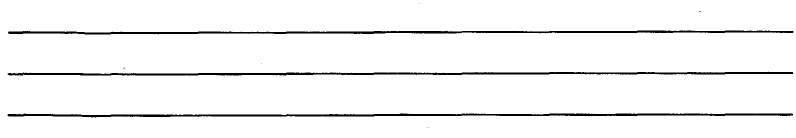

\section{Patterns transactionnels identifiés :}

Fonctionnel

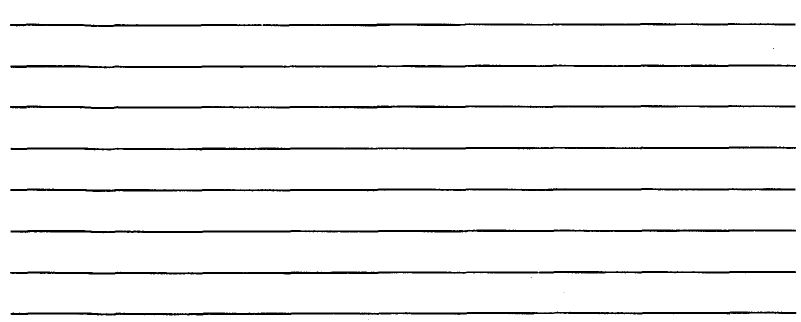

Recomposition :

$$
\text { Loyauté : }
$$

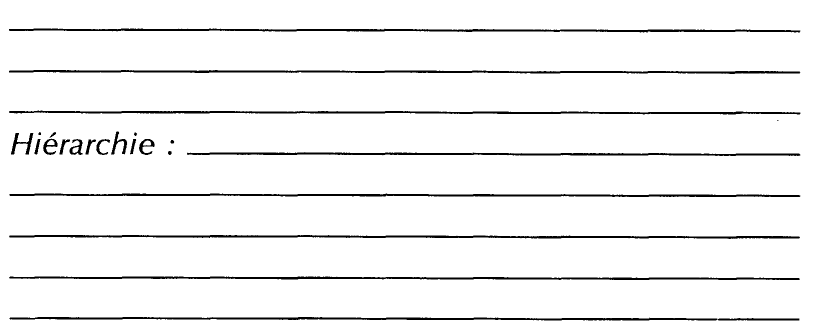

Dysfonctionnel

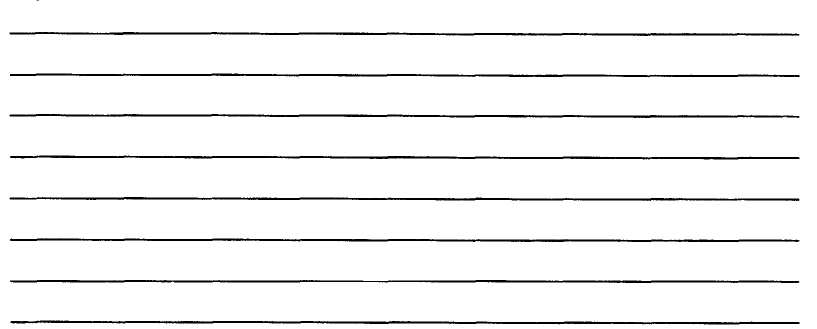




\section{Planification de l'action}

1. Forces du système familial recomposé à utiliser.

2. Faiblesses du système familial recomposé à améliorer.

3. Problème(s) reformulé(s) identifié(s) avec la famille.

4. Objectif général déterminé avec la famille.

5. Changements visés identifiés avec la famille et priorisation.

6. Motivation $(M)$ au changement : degré de malaise $(m)$ et degré d'espoir (e) pour chaque membre.

7. Fonction du problème. 
Il est fréquent, dans une famille recomposée, qu'un enfant appartienne à plus d'un type de sous-systèmes fraternels, tout comme nous retrouvons souvent plus d'un sous-système de même type (ex. : deux sous-systèmes consanguins qui forment alors un sous-système par alliance).

Dans notre grille, pour chaque sous-système fraternel, nous en précisons le type et de quels enfants il est composé. Ensuite, comme principales fonctions, nous retenons l'aspect laboratoire de vie sociale : apprentissage, par la fratrie, du respect des grandes règles de la vie de société, respect des règles du jeu, entente, négociation, compromis, partage des personnes, des objets et des lieux, etc.; la cohésion : capacité de la fratrie de faire front commun, de se liguer positivement ou négativement pour atteindre ensemble un objectif désiré; et le support : capacité de se donner un soutien affectif entre eux, se consoler, se confier mutuellement. La possibilité pour ces soussystèmes de pouvoir accomplir ces fonctions peut varier selon l'âge des enfants. Dans notre famille, notre grille se lit ainsi :

Sous-système fraternel :

type : consanguin - Isabelle et Frédéric

laboratoire de vie sociale : pauvre, relation plus parent-enfant que frère-sœur, avec peu de négociation et de partage égal;

cohésion : parfois négative;

support : Isabelle soutient son frère, rarement l'inverse.

type : par alliance - Isabelle, Frédéric et Julie

laboratoire de vie sociale : bon entre Frédéric et Julie, Isabelle est exclue;

cohésion : parfois entre Frédéric et Julie;

support : bon entre Frédéric et Julie.

Notons que Julie, fille de Paul et Diane, fait partie d'un soussystème fraternel mi-consanguin avec Benjamin, fils de Pierre et Diane; mais celui-ci n'est pas un sous-système de notre famille recomposée, Benjamin ne résidant en aucun temps dans celle-ci. II s'agit donc d'un sous-système fraternel dans l'autre famille recomposée que Diane, mère de Julie, a formée avec Pierre. Julie est membre de deux familles recomposées différentes.

Cela nous amène à tracer une Carte de la famille recomposée et de ses environnements, qui présente toutes les personnes identifiées et les environnements présents et significatifs, et ajoute les ressources à mobiliser. Pour certains éléments de la carte, nous qualifions le type 
de frontières en utilisant ces signes, sur un continuum, pour des frontières

$\overline{\text { désengagées }}-\underset{\text { claires }}{-}-\cdots \cdots \cdots \cdot \cdots \cdot \cdots$

Nous retrouvons donc la carte suivante :

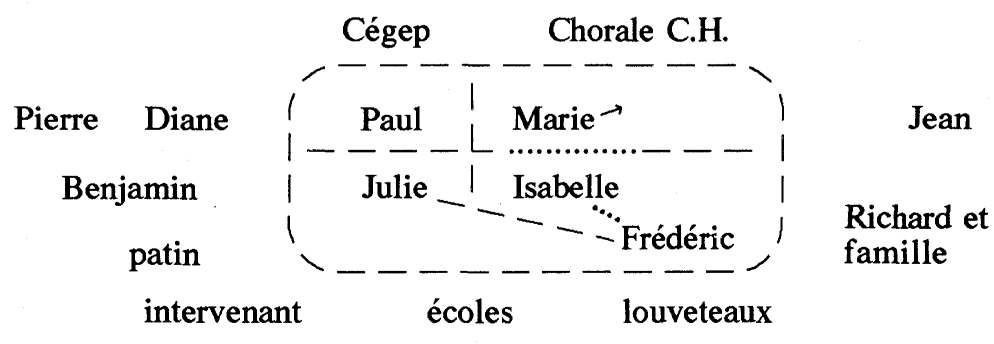

Source : Assoc. des familles recomposées de Québec Métro.

Dans notre travail de formation ou avec les familles, nous avons I'habitude d'utiliser différentes couleurs pour différencier les appartenances biologiques. Si cela avait été possible, dans cet article, nous aurions eu :

\begin{tabular}{lc} 
en bleu, le lien biologique & Pierre Diane \\
en rouge, le lien biologique & Benjamin \\
en vert, le lien biologique & Paul Diane \\
& \multicolumn{2}{c}{ Julie } \\
Jsabelle $\quad$ Marie & Frédéric
\end{tabular}

Nous constatons que certaines personnes ont des couleurs superposées, qui illustrent des relations différentes. Ainsi, il serait aussi facile de repérer d'un seul coup d'œil les appartenances biologiques, de même que les unions antérieures.

Le point séparation nous indique de quand datent les séparations des unions antérieures; le point recomposition, depuis quand la famille est recomposée. Nous constatons ici :

Séparation : Marie -8 ans

Recomposition : 13 mois

$$
\text { Paul }-3 \text { ans }
$$

Ces renseignements juxtaposés donnent des indications sur l'évolution des familles, du nucléaire à la recomposition.

Sous deuil, nous indiquons notre perception de « qui n'a pas fait le deuil de quoi, de qui ». Il pourrait s'agir d'un parent qui n'a pas fait 
le deuil de son conjoint précédent, d'un enfant qui n'a pas fait le deuil de la famille nucléaire ou d'un enfant qui n'a pas fait le deuil de la famille " entre-deux " dans laquelle il avait trouvé un équilibre satisfaisant. Dans notre famille, nous retrouvons :

Deuil : Isabelle n'a pas fait le deuil de la famille monoparentale antérieure qui lui convenait.

Nous évaluons la loyauté sous deux aspects : permission donnée et actualisation. Nous indiquons si le parent donne à l'enfant la permission d'être loyal, d'aimer son autre parent, l'ex-conjoint, et insistons sur la cohérence de cette permission. La permission d'être loyal à l'autre parent ne se résume pas à dire " je sais que c'est normal qu'il aime son père et je l'accepte ", la cohérence doit se manifester dans les propos tenus à son sujet. Si le parent donne, d'une part, la permission verbale à l'enfant d'être loyal et, d'autre part, décrit régulièrement l'autre parent comme un "bon à rien ", il y a manque de cohérence dans la permission donnée. Que cette permission soit accordée ou non, l'enfant fait aussi le choix d'être loyal ou non. Il est important $d^{\prime}$ indiquer sur la grille la permission et sa cohérence et $s^{\prime} i l y$ a loyauté. Dans notre exemple, nous constatons que cet élément ne cause pas de problème :

Loyauté : les permissions d'être loyal sont bien données et chaque enfant exerce cette loyauté.

Pour ce qui est des frontières, déjà la carte familiale nous en donne un aperçu. Ici, nous précisons des frontières difficiles à mettre sur la carte, faisons ressortir des éléments additionnels ou résumons la situation. Nous résumons ici :

Frontières : la majorité des frontières dans la famille sont claires, sauf I'enchevêtrement entre Marie et Isabelle, qui cause parfois de l'enchevêtrement entre Isabelle et Frédéric.

La hiérarchie reflète les positions dans la relation de couple et la relation parent-enfant; le sous-système conjugal doit être égalitaire alors que le parent est en position supérieure par rapport à l'enfant. Dans notre famille, l'enchevêtrement des frontières cause un problème dans la hiérarchie :

Hiérarchie : problème existant déjà dans la famille monoparentale qui se poursuit, Isabelle étant enfant parental.

La deuxième page de la grille se termine avec l'identification des patterns transactionnels, I'un fonctionnel, l'autre dysfonctionnel, toujours pour faciliter la planification de l'intervention. Par patterns transactionnels, nous entendons les façons de faire dans les échanges du 
quotidien, connues de tous et répétitives. Pour notre famille, nous sélectionnons :

\section{Patterns transactionnels identifiés :}

fonctionnel : Frédéric demande

à Marie une sortie, Marie I'accorde, Isabelle manifeste son désaccord, Marie maintient sa décision, Isabelle monte le ton, Paul appuie Marie dans sa décision, Marie accorde la permission à Frédéric de façon claire et dit à Isabelle de se taire, Isabelle bougonne un peu et se tait. dysfonctionnel : Isabelle réprimande Frédéric à la table, Frédéric argumente, le ton monte, Paul rappelle à Marie que c'est à elle de réprimander Frédéric, Marie essaie faiblement, Isabelle dit à Paul de se mêler de ses affaires, le ton monte entre Paul et Isabelle, Marie essaie de les calmer sans succès, Paul sort de la table, " n'a plus faim ».

La troisième page de la grille, dans laquelle nous reconnaissons davantage les éléments de l'approche court terme planifié, amorce l'élaboration du plan d'intervention. Au point 1., Forces du système familial recomposé à utiliser, il s'agit, non pas de reconnaître de nouvelles forces, mais de bien regarder la page précédente de la grille et de regrouper les forces déjà identifiées que l'intervenant utilisera dans son intervention. La famille Lemieux-Laforce a plusieurs forces :

Forces du système familial recomposé à utiliser :

- sous-système conjugal sain et fort;

- capacités de materner et guider de Paul et Marie;

- capacité de contrôler chez Paul;

- loyauté permise et actualisée;

- deuil des familles nucléaires antérieures surmonté;

- les membres, pris individuellement, ont des forces.

Pour les faiblesses du système familial recomposé à améliorer, nous procédons de la même façon. Ces faiblesses à améliorer se retrouveront, dans le plan d'intervention, soit dans les changements visés identifiés avec le client, soit dans l'agenda caché de l'intervenant qui les inclura alors dans les changements visés. Ici, les faiblesses sont :

Faiblesses du système familial recomposé à améliorer :

- faible capacité de contrôler de Marie;

- enchevêtrement de certaines frontières;

— plusieurs éléments des sous-systèmes fraternels.

Le problème reformulé identifié avec la famille donne souvent du «fil à retordre» aux intervenants. En court terme planifié, le 
problème reformulé est le point de départ du contrat thérapeutique et résulte de l'évaluation de l'intervenant. Il peut être différent du problème retenu par le client comme étant le problème principal. Toutefois, il est essentiel que le système-client reconnaisse le problème qu'il a retenu à l'intérieur du problème reformulé. La reformulation du problème doit inclure toutes les personnes qui le vivent et qu'il faut impliquer dans le processus de changement. II doit $y$ avoir consensus du système-client et de l'intervenant sur le problème reformulé. N'oublions pas que le problème se reformule en "difficultés», en termes négatifs. Notre famille retient comme problème principal qu'lsabelle $n^{\prime}$ accepte pas que sa mère se soit mise en couple avec Paul. L'intervenant doit donc proposer un problème reformulé plus large que cette vision du problème retenu, incluant l'ensemble des difficultés vécues par le système familial et identifiant la problématique de la recomposition de la famille. Un consensus s'est dégagé sur la reformulation suivante :

\section{Problème reformulé identifié avec la famille :}

La recomposition de votre famille a provoqué des changements qui amènent différentes frustrations à plusieurs d'entre vous, frustrations que vous n'arrivez pas à résoudre : pour Isabelle, la frustration de ne plus pouvoir exercer l'autorité qu'elle avait auparavant sur Frédéric; pour Paul, d'être l'objet des "flèches " $d^{\prime}$ Isabelle et de voir que Marie se fait mener par Isabelle; pour Marie, d'être coincée entre Paul et Isabelle assez souvent; et pour les autres enfants, de subir le climat tendu qui en résulte.

Le consensus sur le problème reformulé influence l'objectif général déterminé avec la famille, deuxième élément du contrat thérapeutique. Il est l'inverse, en termes positifs, du problème reformulé. Vouloir y ajouter d'autres objectifs généraux serait irrespectueux de l'entente négociée avec le client sur le problème reformulé. Nous retrouvons, à ce point :

\section{Objectif général déterminé avec la famille :}

Trouver des solutions satisfaisantes aux différentes frustrations provoquées par les changements dus à la recomposition de notre famille.

L'élément Changements visés identifiés avec la famille et priorisation constitue le point suivant du contrat thérapeutique. Ces changements visés sont également les objectifs particuliers de l'intervention. Tout comme le problème reformulé et l'objectif général, ils sont le résultat d'un consensus entre l'intervenant et le système-client. Ces changements visés, qui déterminent la planification de l'intervention, doivent être précis, limités et énoncés en termes mesurables, 
travaillables et observables. Ils guideront l'intervenant tout au long de l'actualisation du plan d'intervention. Pour les choisir, nous demandons à la famille quels sont les changements qui devront être mis en place pour que l'objectif général soit considéré comme atteint et le problème reformulé résolu. Nous facilitons cette tâche en faisant bien préciser ces changements que nous limitons à un maximum de cinq. Notre famille a arrêté les changements visés suivants :

\section{Changements visés identifiés avec la famille et priorisation :}

Nous arriverons à résoudre les différentes frustrations provoquées par la recomposition de notre famille quand :

1. nous connaîtrons et appliquerons les règles de fonctionnement idéal pour une famille recomposée;

2. Marie assumera elle-même et de façon constante son autorité parentale auprès de Frédéric;

3. nous échangerons nos points de vue, sans nous chicaner, sur les difficultés inévitables du quotidien;

4. nous aurons tous ensemble des activités de loisirs agréables.

Le contrat thérapeutique comprend quatre autres éléments : les participants, les moyens, les modalités techniques et les engagements respectifs. Nous ne les incluons pas dans cette grille, pour ne pas I'alourdir inutilement.

Par ailleurs, nous avons inclus : Motivation (M) au changement : degré de malaise $(\mathrm{m})$ et degré d'espoir (e) pour chaque membre. Que de fois, devant les piètres résultats d'une intervention, entendons-nous "le client n'était pas motivé ». Une juste évaluation est essentielle pour le plan d'intervention : les efforts de changement demandés seront en relation avec la motivation. Le plus beau plan d'intervention est voué à l'échec si le client n'a pas une véritable motivation au changement. Il ne suffit pas qu'un client se présente à son rendezvous pour conclure qu'il est motivé. Pour évaluer la motivation, nous tenons compte du degré de malaise « $m$ » qui suscite la volonté du client au changement et du degré d'espoir "e » qui reflète la croyance du client dans sa propre capacité de réussir le changement. Il faut prendre note qu'il s'agit de la croyance du client dans sa capacité, non de la croyance de l'intervenant dans la capacité du client. Malaise et espoir doivent être assez grands pour qu'il y ait une véritable motivation au changement. Pour notre famille, nous avons une bonne motivation. La grille montre :

Motivation au changement : degré de malaise $(m)$ et degré d'espoir (e) pour chaque membre

Paul

Marie Isabelle grand $m$ et grand $e$ grand $m$ et grand $e$ moyen $\mathrm{m}$ et grand $\mathrm{e}$ bonne motivation bonne motivation motivation moyenne 
Bien que Frédéric et Julie soient considérés dans l'intervention, leur degré de motivation au changement n'a pas besoin d'être grand, puisqu'ils ressentent moins de frustrations que les autres membres de la famille. Leur collaboration est sollicitée.

Enfin, notre grille se termine avec la fonction du problème, un point difficile à trouver. Ici, nous émettons une hypothèse sur la fonction que remplit le problème dans le fonctionnement de la famille. Pour y arriver, nous répondons à la question « à qui ou à quoi sert le problème?" et cherchons une réponse positive. Dans l'approche systémique, la fonction du problème est une solution, bien qu'inadéquate, face à une menace, qui réussit à maintenir l'équilibre du système, mais de façon insatisfaisante pour le développement des membres. Par ailleurs, nous savons que les difficultés vécues par un système ne remplissent pas toujours une telle fonction. Ainsi il est normal qu'en réorganisation un système éprouve des difficultés d'adaptation. Dans notre famille, la recomposition s'est faite il y a 13 mois. Les conflits actuels démontrent des difficultés plus importantes que celles d'un système en réorganisation, empêchant l'évolution normale de la famille recomposée dans la poursuite d'un équilibre satisfaisant. La fonction du problème nous apparaît être :

Fonction du problème : le problème sert à Isabelle, dans la relation fille-mère, à préserver les gains que sa mère lui accordait dans la famille monoparentale d'avant et à continuer d'aider sa mère dans une fonction difficile pour cette dernière. II sert aussi à ce que Marie maintienne quelques-uns des patterns vécus durant sept ans, et ressente moins de culpabilité face à la perte d'importance et de pouvoir que son remariage a entraînée pour sa fille.

L'intervenant devra donc avoir un certain agenda secret où il veillera à ce qu'Isabelle puisse obtenir d'autres gains et être rassurée sur les capacités parentales de sa mère dans le nouvel équilibre atteint à la fin de l'intervention. II devra aussi vérifier si Marie est suffisamment rassurée par les progrès de sa fille pour accepter de liquider les vestiges des patterns de la période de transition entre les deux unions, de même que son sentiment de culpabilité.

\section{Les principales attitudes et techniques}

Dans cette dernière partie, nous voulons présenter brièvement les principales attitudes et quelques techniques utilisées pour recueillir les données de cette grille. Ce sont celles de l'approche systémique planifiée à court terme, avec certaines adaptations reliées aux caractéristiques des familles recomposées.

Notre attitude fondamentale est 1 . de considérer la situation vécue par la famille de façon positive, par une «normalisation » de ce 
qui se passe; 2 . de voir les membres de la famille comme les responsables du changement. Notre responsabilité comme intervenants est de les guider et de leur apporter notre soutien.

Nous insistons sur le fait que la famille vit une difficulté qui peut être reliée à l'obligation qu'elle a d'apprendre une façon de vivre différente de ce à quoi ses membres ont été habitués durant une période de temps. Nous insistons sur l'intention positive de chacun. Nous travaillons davantage sur des apprentissages et de l'évolution en fonction de problèmes.

Tout en nous centrant sur le vécu propre à la famille, nous commençons à " dépsychologiser » et à " défamilialiser " la perception qu'ont les membres de leurs problèmes, en faisant ressortir le caractère social de la situation, et les influences de divers environnements sur leur organisation de vie et sur les réactions des membres.

En même temps, nous procédons avec beaucoup de prudence, car la plupart des familles ne consultent que lorsque les problèmes sont rendus très importants et que certains membres ont pris une position rigide, défensive ou coercitive, allant jusqu'à viser l'expulsion d'un des membres, qu'il s'agisse d'un enfant ou du beau-parent.

Nous travaillons généralement avec toute la famille et même avec des personnes externes mais significatives, durant la phase d'exploration. Nous utilisons aussi des rencontres avec les sous-systèmes, de façon souple et d'après ce que la situation semble demander.

Enfin, une de nos principales préoccupations est d'arriver rapidement, grâce à l'utilisation de la grille, à un diagnostic différentiel qui distingue ce qui est relié à la recomposition de la famille, ce qui dépend des sous-systèmes, surtout conjugal, et ce qui relève des personnes, de leurs apprentissages et de leurs capacités insuffisamment développées pour l'exécution de leurs rôles. Cela nous amène à décider qui participera aux rencontres suivantes.

Pour effectuer cette démarche, nous utilisons les techniques régulières de l'intervention systémique planifiée à court terme, avec des modifications reliées à la problématique. Nous prenons un temps plus long pour joindre tous les membres, en écoutant chacun, surtout les enfants; nous demandons à ces derniers de clarifier qui est relié à qui, et comment. Cela nous permet de déterminer, au-delà des liens de consanguinité, qui a des liens harmonieux avec qui, qui est en marge, qui est mis à l'écart, et comment les autres réagissent lorsque ces positions sont clarifiées.

Nous utilisons les "six questions" (voir annexe) de façon systématique, en faisant parler tous les membres lorsque c'est possible, et en les faisant réagir aux réponses des autres. Lorsque la relation de confiance est assez établie entre nous, nous devenons 
ouvertement actifs et directifs. Il s'agit de faire reconnaître et préciser les faits, les conditions de vie, les comportements et les transactions qui les entourent. Pour cela, nous nous servons des différentes sortes de questions. Mais notre but étant de connaître l'organisation de vie, l'évolution qui a conduit à la situation présentée et les conditions qui la maintiennent, nous amenons les membres à interagir, et nous observons les réactions des autres. Nous pouvons alors constater si des étapes n'ont pas été réalisées, ou si elles ont été sautées; nous pouvons aussi constater si des membres refusent les nouvelles organisations de relations ou d'autorité. Et nous pouvons vérifier qui réagit de façon positive à la proposition de solutions possibles, c'està-dire qui est probablement le plus prêt à s'engager dans un changement.

Nous sommes très attentifs à ce que les membres disent sur les parents absents, qui en parle et comment, et quelles sont les réactions dites et non dites des autres. Nous veillons à ne pas donner l'impression de prendre parti pour ou contre eux, quelles que soient les situations qui sont présentées. Les problèmes présentés par les " ex» constituent une des principales sources d'échec des familles recomposées : les conjoints doivent régulièrement négocier comme paire exécutive, alors que certaines décisions sont déjà prises en grande partie dans la paire exécutive des parents. Le conjoint non parent se sent alors facilement agressé dans son intimité de couple, et dépossédé de l'attention et du pouvoir qui lui reviendrait dans une famille non recomposée. Les interventions de correction - si possible - de cette dynamique n'entrant pas dans le cadre de cet article, nous ne pouvons approfondir davantage cette question ici.

Nous sommes attentifs à l'identification des alliances exagérées, surtout transgénérationnelles, qui peuvent être le vestige de la période entre la séparation et la recomposition. La relation entre Isabelle et sa mère dans le cas présenté plus haut est un bon exemple. Nous cherchons aussi s'il y a eu des unions temporaires durant cette période, et quelles ont été les conséquences sur le fonctionnement des membres, surtout dans les relations entre les enfants et le conjoint non parent ou dans le développement de sentiments de culpabilité exagérée chez le parent.

Nous utilisons les questions circulaires telles que développées par Karl Tomm ${ }^{7}$ et $\mathrm{F}$. Roestler ${ }^{8}$, non seulement pour connaître les alliances et les coalitions, les oppositions secrètes et la hiérarchie, mais surtout pour amener les membres à reconnaître les conséquences systémiques de leurs comportements, et à réfléchir en présence des autres sur leurs enjeux réels. Notre but est de les engager dès cette première rencontre dans un processus de changement commun. 
Cette activité est accompagnée d'un support actif, où nous établissons clairement des règles nouvelles, qui donnent la permission aux membres de parler et d'expérimenter des façons différentes de se relier.

Dès la première rencontre, une fois que nous avons pu préciser les relations et l'organisation de la famille, nous adoptons un rôle d'enseignant, pour offrir des solutions nouvelles, en soulignant que, de toute façon, ils réussissent déjà - souvent ou parfois — à avoir de bons moments ensemble. L'approche n'est pas surtout cognitive, car nous insistons plus sur l'expérimentation que sur l'explication. Nous leur faisons expérimenter des échanges positifs, et nous en faisons ensuite valoir les conséquences ou la signification pour leur organisation de vie. S'ils ne comprennent pas, mais changent, c'est bien; s'ils changent et comprennent, $c^{\prime}$ est mieux.

$S^{\prime}$ ils peuvent reconnaître les transactions fonctionnelles et dysfonctionnelles, et leur impact sur le développement de la famille et des personnes, les changements auront plus de chances d'être durables et d'avoir des répercussions dans les autres zones de vie.

Par exemple, quand les enfants entendent des commentaires ouverts sur l'importance de la complémentarité des conjoints comme paire exécutive, ils voient les activités du conjoint du parent sous un autre jour. L'accent que nous mettons sur l'importance des périodes entre la séparation et la recomposition a souvent amené des adolescents à dire qu'ils comprenaient mieux leurs difficultés à laisser leur position et leur rôle auprès de leur parent. L'aspect peut-être le plus frappant est la réaction des enfants quand nous clarifions la valeur et même la nécessité de la loyauté envers le parent absent : cette permission apporte d'abord un soulagement, puis des expressions parfois très profondes de sentiments. II peut $y$ avoir ensuite des tensions avec le parent ou le conjoint; ces tensions devraient de toute façon être travaillées.

Nous insistons sur ce point : les enfants savent ce qui se passe dans leur famille : ils réfléchissent et se sentent impliqués, quelles que soient leurs réactions. Une fois que des règles nouvelles ont été établies sur ce qui peut être dit, que nous avons commencé à préciser l'impact des transactions et que nous amenons les enfants à s'exprimer, nous sommes régulièrement abasourdis et émerveillés par la qualité et la profondeur de leurs commentaires sur le vécu de la famille, leurs réactions et leurs désirs d'amélioration. Et cela, dès 7 ou 8 ans. Cet apport, venant de l'intérieur de la famille, constitue parfois le facteur provoquant le changement. 
LES 6 QUESTIONS*

\begin{tabular}{|c|c|}
\hline Questions & Commentaires \\
\hline $\begin{array}{l}\text { 1. Parmi les problèmes, quel est le problème } \\
\text { principal qui vous préoccupe présen- } \\
\text { tement? }\end{array}$ & $\begin{array}{l}\text { Cette question sert à clarifier les ambiguïtés et ambivalences de début et à } \\
\text { dissiper la confusion entre la situation générale, les multiples problèmes } \\
\text { énumérés et le problème ressenti au moment présent. }\end{array}$ \\
\hline $\begin{array}{l}\text { 2. Quel est l'impact de ce problème sur vous } \\
\text { présentement? } \\
\text { Et s'il ne se règle pas? }\end{array}$ & $\begin{array}{l}\text { Cette question aide le client à assumer le problème, en se plaçant dans la } \\
\text { situation au lieu de se voir comme spectateur. Elle aide à mesurer le malaise et } \\
\text { l'espoir ressenti par le client. }\end{array}$ \\
\hline $\begin{array}{l}\text { 3. Aidez-moi à mieux comprendre. } \\
\text { a) Comment le problème s'est-il manifesté } \\
\text { pour vous? } \\
\text { b) Qu'est-ce qui se passe présentement? } \\
\text { c) Qu'est-ce qui arrivera dans I'avenir } \\
\text { d'après vos précisions? }\end{array}$ & $\begin{array}{l}\text { On explore le passé en relation avec le présent; on décortique le présent; on } \\
\text { cherche les éléments structurels qui influent sur le problème et on mesure le } \\
\text { sentiment de malaise. On commence immédiatement à l'augmenter. Enfin, } \\
\text { cette question identifie les patterns transactionnels, si nécessaire, en amenant } \\
\text { le client à considérer les inconvénients probables à venir si la situation ne } \\
\text { s'améliore pas. }\end{array}$ \\
\hline $\begin{array}{l}\text { 4. Qu'avez-vous fait pour régler le } \\
\text { problème? }\end{array}$ & $\begin{array}{l}\text { Cette question aide à éviter les « oui mais » et fait éviter aussi de proposer des } \\
\text { actions qui ont déjà été posées; elle permet de renforcer et de valider les } \\
\text { tentatives positives déjà faites. Elle renseigne enfin sur le degré de motivation à } \\
\text { vouloir régler le problème. }\end{array}$ \\
\hline $\begin{array}{l}\text { 5. Quelles étaient les autres actions possibles } \\
\text { que vous avez considérées ? } \\
\text { Si une action qui semblait possible n'a pas } \\
\text { été posée, qu'est-ce qui vous en a empê- } \\
\text { ché ? } \\
\text { Si vous avez fait des démarches, quels en } \\
\text { ont été les résultats? }\end{array}$ & $\begin{array}{l}\text { Ces questions facilitent l'opérationnalisation de certaines actions possibles, } \\
\text { permettent la prise de conscience de l'influence d'éléments du milieu et } \\
\text { aident à connaître les ressources utilisables ainsi que les peurs et les craintes } \\
\text { qui font obstacle. Elles renseignent sur les facteurs d'espoir : connaissance } \\
\text { des moyens, accès aux ressources, etc. }\end{array}$ \\
\hline $\begin{array}{l}\text { 6. Et maintenant comment pensez-vous que } \\
\text { je puisse vous être utile? }\end{array}$ & $\begin{array}{l}\text { Cette question sert à clarifier les attentes du client. Elle aide aussi l'intervenant } \\
\text { à répondre à ce qui est demandé (on donne parfois trop ou à côté), à suggérer } \\
\text { d'autres solutions qui peuvent être offertes et à clarifier le rôle de l'aidant. }\end{array}$ \\
\hline
\end{tabular}

* Élaine Carey-Bélanger et Christian Côté, Service social, été 82, "Les interventions brèves : 8 ans après ", p. 259-260; révision avec Denise Robitaille. Une recherche réalisée par C. Côté avec 3 groupes d'étudiants en stage dans des CLSC de 1982 à 1985, auprès de 75 clients, établit que l'utilisation rigoureuse de ces six questions a été suivie de changements significatifs dans $83 \%$ des cas. 


\section{Conclusion}

Nous avons voulu présenter cet article, beaucoup plus technique qu'analytique, parce que nous croyons que la grille et ses modalités

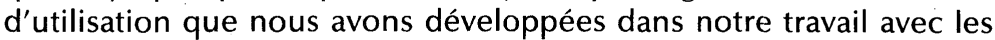
familles recomposées peuvent faciliter la tâche de plusieurs intervenants, en réorganisant de façon structurée les informations recueillies à l'exploration et en permettant ainsi de parvenir à une planification plus précise. Bien qu'elle puisse sembler complexe, elle se révèle simple d'emploi, si on l'applique de façon rigoureuse, sans se laisser aspirer par les éléments du contenu émotif. Nous avons pu constater, dans nos interventions directes auprès des familles et par les commentaires des intervenants sociaux qui l'ont utilisée dans les formations données au Centre, qu'elle est une source de sécurité et de plus grande efficacité. Elle ne remplace pas les attitudes de base dans l'intervention, elle les encadre et les soutient. En même temps, nous savons qu'elle est sujette à des ajustements et à des améliorations, pour la rendre encore plus précise tout en conservant sa simplicité. C'est pourquoi nous sommes intéressés à connaître les résultats des expérimentations des lecteurs.

\section{Notes}

1 Service social, été 1974, numéro spécial sur l'intervention planifiée à court terme. Élaine Carey-Bélanger et Christian Côté, Service social, été 1982, "Les interventions brèves : 8 ans après ". Mathilde du Ranquet, Les approches en service social, Edisem, 1991.

${ }^{2}$ Familles en thérapie, France-Amérique, 1974. Voir la présentation des concepts dans Barbara DeFranck-Lynch, Thérapie familiale structurale, ESF, 1987.

${ }^{3}$ Jay Haley, Nouvelles stratégies en thérapie familiale, France-Amérique, 1976. Cloé Madanès, Derrière la glace sans tain, ESF, 1981; Stragetic Family Therapy, Guilford, 1978.

${ }^{4}$ La thérapie avec la famille, ESF, 1981; Behind the Family Mask, BrunnerMazel, 1983.

${ }^{5}$ Invisible Loyalties, Harper, 1973. Voir en français M. Heireman, Du côté de chez soi : la thérapie contextuelle de Boszormenyi-Nagy, ESF, 1989.

${ }^{6}$ Cette phrase nous a été dite par plusieurs enfants, devant les conjoints.

${ }^{7}$ Karl Tomm, Fam. Process, mars 1987; juin 1987 et mars 1988 : Interventive Interviewing : Part I-II-III : "On Strategic, Reflexive and Circular Questionning ".

${ }^{8}$ F. Roestler, Présentation au congrès du Child Guidance Clinic, de Philadelphie, novembre 1987. 